\title{
Distribution of multiband THz wireless signals over fiber
}

\author{
Haymen Shams", Luis Gonzalez-Guerrero, Martyn Fice, Zhen Yang, Cyril Renaud and Alwyn Seeds \\ Department of Electronic and Electrical Engineering, University College London, Torrington Place, \\ London, WC1E 7JE, UK. \\ *h.shams@ucl.ac.uk
}

\begin{abstract}
Terahertz wireless communication is receiving great interest from researchers and industries, thanks to the new spectral windows between 0.1 and $1 \mathrm{THz}$ offering opportunities for ultra-high-data-rate wireless transmission. Wavelength division multiplexing for wireless-over-fiber is foreseen as an enabling technique to support connection between base stations and a central station. This paper reviews architectures for photonic distribution and generation of multiband signals for sub$\mathrm{THz}$ wireless communications, giving rates up to $100 \mathrm{~Gb} / \mathrm{s}(20 \mathrm{~Gb} / \mathrm{s}$ per band) using the full spectrum between $220 \mathrm{GHz}$ and $280 \mathrm{GHz}$ for downlink wireless transmission, and $10 \mathrm{~Gb} / \mathrm{s}$ for uplink using on-off keying.
\end{abstract}

Keywords: Microwave photonics, millimeter $(\mathrm{mm})$-wave generation, optical heterodyne, wireless over fiber, $\mathrm{THz}$ wireless communication.

\section{INTRODUCTION}

Higher capacity wireless access networks are desired to serve growing demands for increasing mobile traffic and multimedia services. Edholm has predicted the evolution of data rates by wireless system is being doubled every 18 months [1]. For example, the global mobile data traffic climbed to 3.7 exabytes/month in 2015 [2]. This continuous growth arises from the end users' needs for higher data rates for potential applications such as HDTV, high quality video conferences, and 3D display. Therefore, wireless communication networks are facing challenges to meet the data traffic demands. Recently, terahertz wireless communication has been receiving great interest from researchers and industry. Frequencies between 0.1 and $1 \mathrm{THz}$ offer opportunities for ultra-high-data-rate wireless transmission. These bands are wide enough and, those above $300 \mathrm{GHz}$, are not yet allocated [3]. However, these mm-wave and THz frequencies are limited by the high free space path loss (FSPL) and atmospheric absorption peaks due to water vapour molecules. Figure 1 (a) shows the specific attenuation due to atmospheric absorption molecules, and FSPL at $10 \mathrm{~m}$ and $100 \mathrm{~m}$. The FSPL attenuates the signal with the square of the carrier frequency, and the molecular absorptions by water vapour confine the transmittable spectrum into several windows. Due to the limited transmission distance, wireless-over-fiber technology can be used to enable the distribution of the $\mathrm{THz}$ signals over a long distance due to the low losses in the optical fiber cables. Many solutions have been introduced to show the synergy between $\mathrm{THz}$ and optical fiber communication for fixed links in outdoors and inside many buildings such as office blocks, shopping malls, and airport terminals. Depending on the link distance, $\mathrm{THz}$ frequency bands can support many potential applications for communications (Figure 1 (b)), including wireless front and backhauling systems, wireless personal area networks in smart homes, kiosk downloads, and device-todevice communications.

Wireless THz generation and detection methods have been developed over several years. The main objective is to obtain high quality $\mathrm{THz}$ signals with high output power necessary for radio communication and other $\mathrm{THz}$ applications. Microwave photonics plays an important role for providing high quality, tunable, and broad bandwidth $\mathrm{THz}$ sources. This method is based on photomixing of two optical tones on a high-speed photodiode. There has been extensive work demonstrated for microwave photonics at lower frequencies in radio-over-fiber networks, due to its simplicity, and this can be easily integrated with the existing optical network infrastructure. In the $\mathrm{THz}$ range, a uni-travelling photodiode 


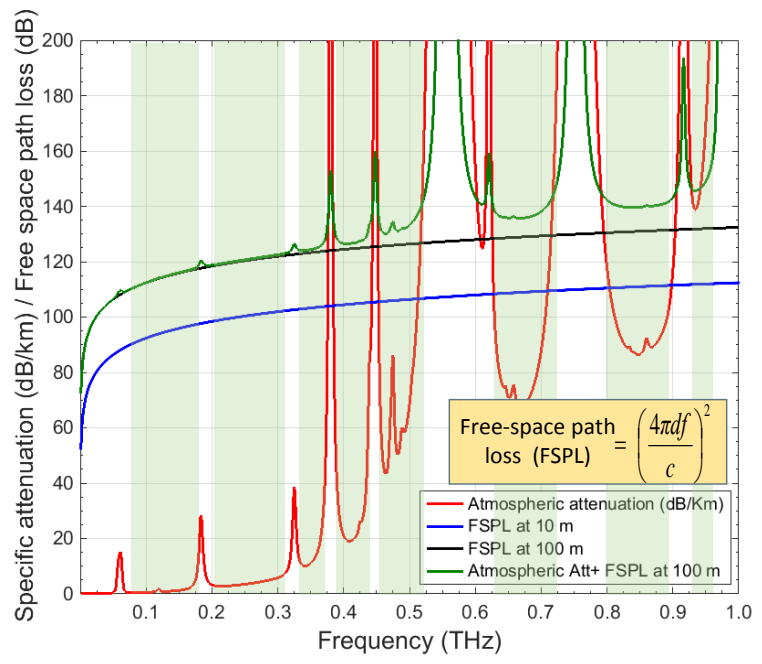

(a)

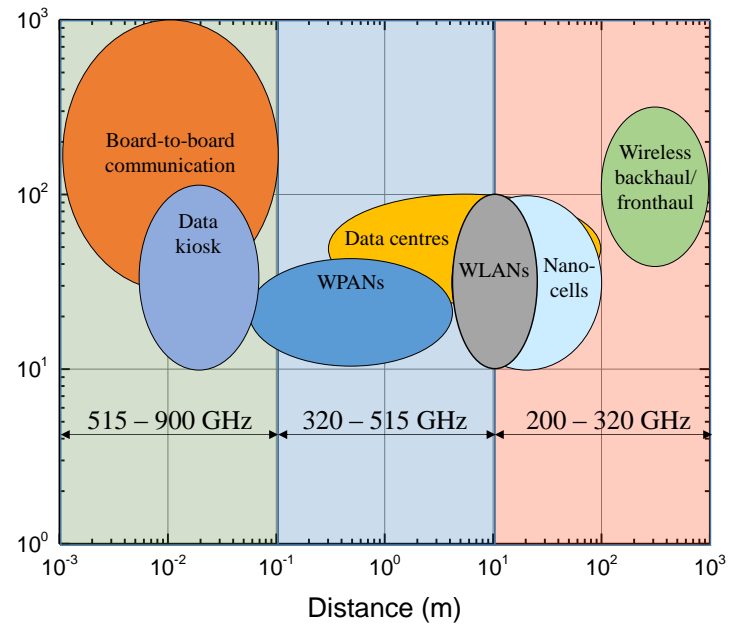

(b)

Figure 1. (a) Specific attenuation due to atmospheric gases based on calculated model ITU-R P676-10 on THz spectrum (0.1- 1 THz), and FSPL at $10 \mathrm{~m}$ and $100 \mathrm{~m}$, and (b) future $\mathrm{THz}$ wireless applications.

(UTC-PD) based on InP is the key element in generating THz signals, which can provide moderate output power even at frequencies $>1 \mathrm{THz}$ [4], [5]. A radiated power of $2.3 \mu \mathrm{W}$ at $1.04 \mathrm{THz}$ for an injected optical power of $430 \mathrm{~mW}$ was achieved with a UTC-PD integrated with a broadband log periodic antenna [6], while a UTC-PD integrated with a transverse electromagnetic horn antenna (TEM-HA) showed $1.1 \mu \mathrm{W}$ at $940 \mathrm{GHz}$ for a photocurrent of only $2.75 \mathrm{~mA}$ and $50 \mathrm{~mW}$ optical power [5]. On the other hand, the combination of the travelling wave (TW) structure with the UTC scheme, offers an enhancement both in the radiated output power and bandwidth. When the TW-UTC-PD is integrated with a resonant antenna, $24 \mu \mathrm{W}$ at $914 \mathrm{GHz}$ can be generated for an optical power of $100 \mathrm{~mW}$ [7]. This device showed a $3 \mathrm{~dB}$ bandwidth of $108 \mathrm{GHz}$, with a maximum power of $1 \mathrm{~mW}$ radiated at $200 \mathrm{GHz}$ [8].

Currently, the IEEE 802.15.3 task group 3d is developing standards for $100 \mathrm{~Gb} / \mathrm{s}$ transmission over ranges from a few centimeters to $200 \mathrm{~m}$ in the $252-325 \mathrm{GHz}$ band, addressing applications from intra-device communications to wireless backhaul. Wavelength division multiplexing for wireless-over-fibre is foreseen as an enabling technique to support connection between base stations and a central station. This would allow the reach of future mm-wave wireless networks to be extended, and enable ease of maintenance and cost-effectiveness goals to be met.

In this paper, we review architectures for photonic generation and distribution of multiband signals for sub-THz wireless communications, demonstrating rates up to $100 \mathrm{~Gb} / \mathrm{s}(20 \mathrm{~Gb} / \mathrm{s}$ in each band) using the full spectrum between 220 and 280 $\mathrm{GHz}$ for downlink wireless transmission, and $10 \mathrm{~Gb} / \mathrm{s}$ for uplink using on-off keying. The sub-THz signals are intended for short-range applications, such as indoor links to mobile units. The use of multiple bands increases the total transmitted data rate while reducing the bandwidth requirements on opto-electronic devices and electronic devices such as the second harmonic mixer in the receiver. The linewidth tolerance is studied numerically to provide a guide to performance with different modulation formats.

\section{PHOTONIC THZ-WIRELESS LINK SYSTEMS}

Several photonic wireless links based on direct or heterodyne detection have been demonstrated in the W-band (75-110 $\mathrm{GHz}$ ) and at higher frequencies. These wireless links can be divided into real-time and offline digital signal processing (DSP) systems. Typically, in a real-time system, an optical source is amplitude-modulated with on-off keyed (OOK) signals. Then, the output is optically heterodyned at the UTC-PD with an unmodulated optical mode at a wavelength separation corresponding to the desired wireless frequency. At the mobile unit (MU), the THz receiver is normally based on a Schottky barrier diode (SBD) used in a direct detection mode, where the amplitude-modulated $\mathrm{THz}$ signal is squarelaw detected and then filtered before performing error counting. This approach is cost effective and simple to implement, 
but suffers from low sensitivity. In the first real-time THz transmission link to be reported, a signal at a carrier frequency of $120 \mathrm{GHz}$ and a data rate of $10 \mathrm{Gbps}$ was transmitted over $1 \mathrm{~km}$ using amplitude shift keying (ASK) [9]. The system was intended for wireless backhaul applications, which can be of key importance in situations where the deployment of optical fiber may not be possible, such as across rivers and difficult-to-access terrains.

In the case of systems employing offline DSP, the SBD is used as a harmonic mixer to downconvert the received THz signal to an intermediate frequency (IF). The IF signal is then sampled in an analog-to-digital converter (ADC) before being processed offline using DSP. The coherent detection offers higher sensitivity performance and the DSP processing can compensate for impairments such as non-linearities and fiber-induced chromatic dispersion. A schematic setup showing a typical coherent $\mathrm{THz}$ system in a single channel configuration is shown in Figure 2. At the MU, a digitized signal is processed according to the following steps: digital downconversion to baseband, resampling, blind equalization using the constant modulus algorithm (CMA), frequency-offset estimation, and then finally carrier phase estimation before bit error detection [10]. In order to increase the channel capacity, higher level modulation formats and polarization multiplexing schemes have also been investigated. By using one of these techniques or both, the spectral efficiency of the system can be improved. However, the improvement requires a coherent receiver, as well as off-line DSP to evaluate the system performance. Transmission of $20 \mathrm{Gbit} / \mathrm{s}$ quadrature phase shift keying (QPSK) and $40 \mathrm{Gbit} / \mathrm{s} 16$ quadrature amplitude modulation (16-QAM) have been demonstrated with an spectral efficiency of $1 \mathrm{bit} / \mathrm{s} / \mathrm{Hz}$ and $2 \mathrm{bit} / \mathrm{s} / \mathrm{Hz}$, over a very short wireless transmission distance, respectively [11], [12]. Moreover, using antenna polarization diversity techniques and optical polarization division multiplexing (PDM) 16QAM, the transmission capacity of a single frequency channel can reach up to $100 \mathrm{Gbit} / \mathrm{s}$ (with spectral efficiency $4 \mathrm{bits} / \mathrm{s} / \mathrm{Hz}$ ) [13].

The most basic arrangement of a photonic THz system uses two free-running lasers as optical sources. Although this approach simplifies the transmitter design and offer great advantage in terms of tuneability and channel allocation, the resultant $\mathrm{THz}$ signal exhibits phase noise resulting from the linewidth and relative frequency fluctuation of the two laser sources. The used of DSP algorithms can compensate this effect at the receiver by applying frequency and phase noise estimation. However, the use of these algorithms comes at the cost of system performance. This depends not only in the linewidth of the two optical sources and the modulation format but also in the specific algorithm used for carrier recovery.

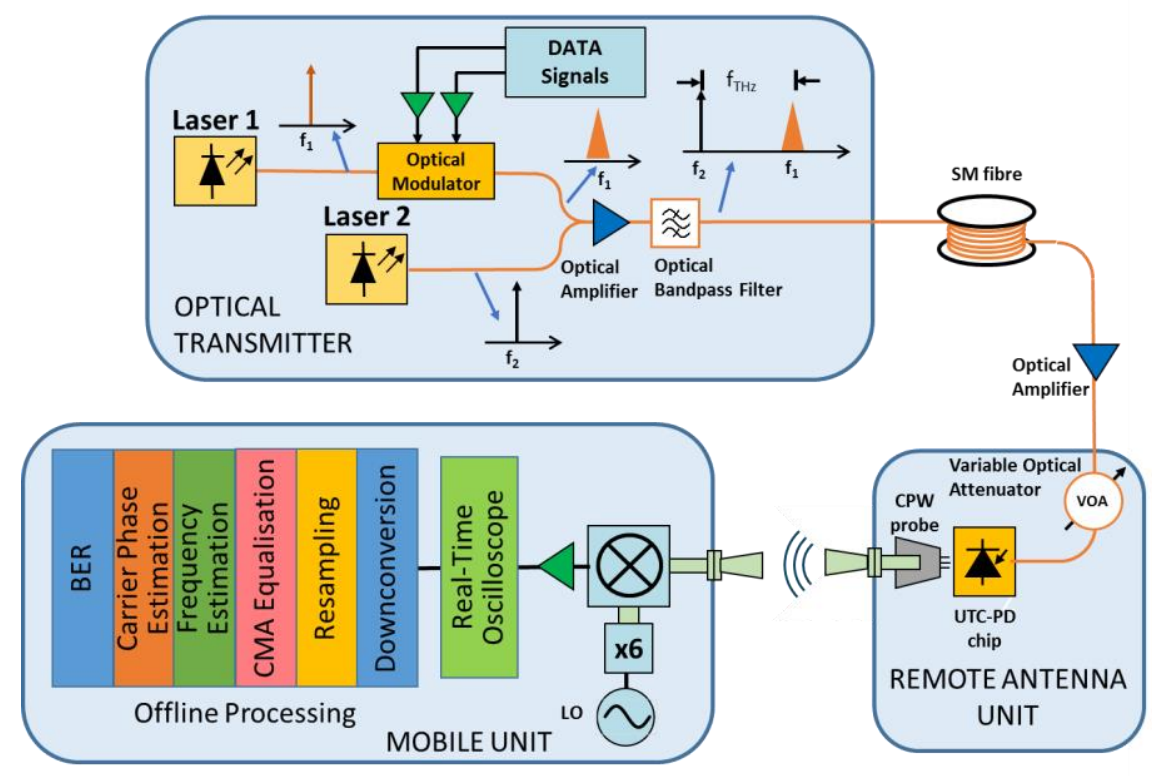

(a)

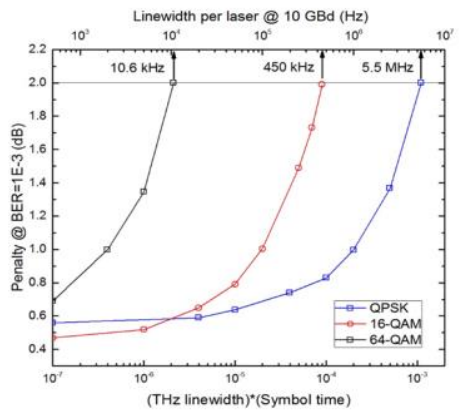

(b)

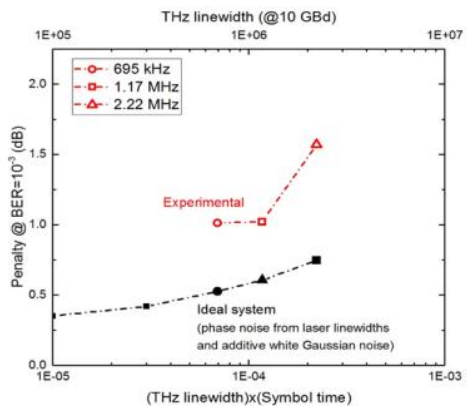

(c)

Figure 2. (a) Schematic of photonic generation of $\mathrm{THz}$ wireless based on two free running lasers, (b) power penalty versus $\mathrm{THz}$ linewidth-symbol time product using Monte Carlo simulations, and (c) power penalty at a BER level of $10^{-3}$ for the simulated ideal system and the experimental setup. 
A commonly used phase estimation algorithm comprises the $M^{\text {th }}$-power operation (Viterbi-Viterbi algorithm) combined with a block-averaging digital filter to remove the distortions from additive white Gaussian noise (AWGN). The power penalty associated with this algorithm versus the THz linewidth-symbol time product is plotted using Monte-Carlo simulation in Figure 2 (b) for different modulation formats [14]. One can see how the penalty increases with both the $\mathrm{THz}$ linewidth, and the number of constellation points in the modulation format. This penalty will limit the maximum laser linewidths that can be used for a given BER performance. The results obtained from the Monte-Carlo (MC) simulations are summarized in Table 1, showing the required laser linewidth for a penalty of $2 \mathrm{~dB}$ at a BER of $10^{-3}$ is $5.5 \mathrm{MHz}, 450 \mathrm{kHz}$, and $11 \mathrm{kHz}$ for QPSK, 16 - and 64 -QAM, respectively. The power penalty associated with different linewidths was also studied experimentally for 10 Gbaud QPSK signals by varying the linewidth of the optical LO. The experimental results (Figure 2 (c)) showed a penalty of less than $2 \mathrm{~dB}$ for a combined linewidth of the both lasers of $2.22 \mathrm{MHz}$ compared to a system without phase noise.

TABLE 1 . THz linewidth requirements for a receiver penalty of $2 \mathrm{~dB} @ \mathrm{BER}$ OF $10^{-3}$

\begin{tabular}{l|l|l|l}
\hline \hline & QPSK & 16-QAM & 64-QAM \\
\hline \hline (THz linewidth)*(Symbol time) & $1 \mathrm{E}-3$ & $9.4 \mathrm{E}-5$ & $2.5 \mathrm{E}-6$ \\
\hline THz linewidth @ 10 Gbaud & $11 \mathrm{MHz}$ & $900 \mathrm{kHz}$ & $22 \mathrm{kHz}$ \\
\hline Linewidth per laser & $5.5 \mathrm{MHz}$ & $450 \mathrm{kHz}$ & $11 \mathrm{kHz}$ \\
\hline
\end{tabular}

\section{PHOTONIC DISTRIBUTION OF MULTIBAND THZ SIGNALS}

The THz spectral band in the range of $200-300 \mathrm{GHz}$ is attracting a strong interest due to its large available bandwidth $>100 \mathrm{GHz}$ with low atmospheric transmission losses. IEEE 802.15 for WPAN is now exploring the frequency band (252 - $325 \mathrm{GHz}$ ) for maximum transmission rate of $100 \mathrm{Gbit} / \mathrm{s}$ [15]. Their prospect for the bandwidth utilization is expected to either use the full bandwidth of $73 \mathrm{GHz}$ for one communication link, or split the spectrum into a several sub-channels with smaller channel bandwidths for one or several communication links. The second case lowers the requirement on the hardware implementation and is beneficial for reducing the signal processing at the receiver. A few experiments have been demonstrated based on a multicarrier system with optical subcarriers at the W-band and beyond $100 \mathrm{GHz}$ to maximize the overall channel data rate, and to achieve high spectral efficiency. We have demonstrated transmission of $75 \mathrm{Gbit} / \mathrm{s}$ in a multichannel system at $200 \mathrm{GHz}$ carrier frequency as illustrated in Figure 3 with closely spaced optical carriers and using a free running laser as an LO for heterodyne mixing [16]. However, the digital signal processing is needed at the receiver

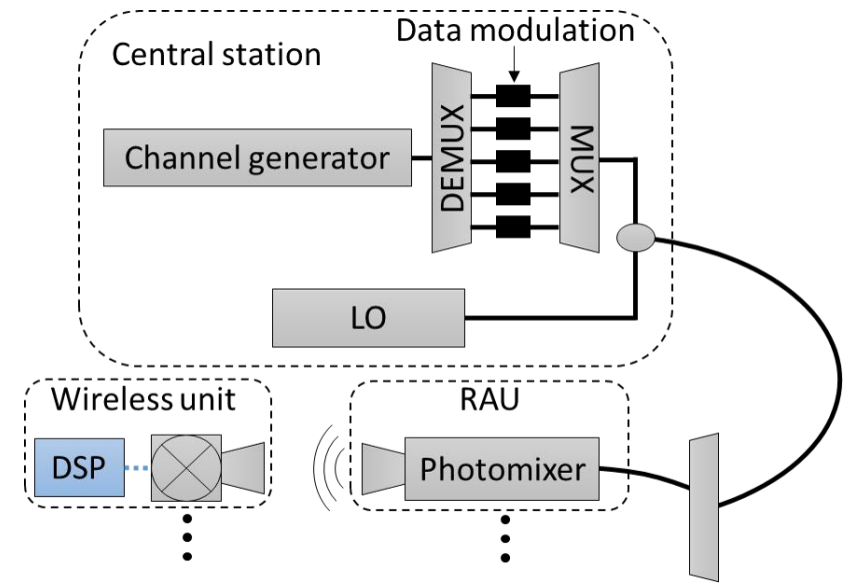

Figure 3. Schematic of photonic multichannel wireless THz distribution. DEMUX and MUX are optical demultiplexer and multiplexer, respectively, DSP is digital signal processing module, and RAU is remote antenna unit. 
to perform a carrier recovery and a phase noise correction, which increases the processing time, complexity, and the system power consumption.

In order to generate high spectral purity modulated $\mathrm{THz}$ signals, the optical frequency comb source was used as a solution to stabilize the frequency fluctuations and the phase noise. The generated THz wireless signals are obtained by heterodyne mixing of two phase correlated optical tones generated from the same source. In [17], we have experimentally demonstrated the photonic generation of multicarrier $\mathrm{THz}$ wireless signals at data rates of up to $100 \mathrm{Gbit} / \mathrm{s}$ using an external injected gain-switched optical comb source shown in Figure 4 (a). The external injected gain switched optical comb source produces phase correlated optical comb lines spaced by the frequency of the driving RF signal. A wavelength selective switch was used in the setup to select a set of three or four optical subcarriers to be modulated with 8, 10 or 12.5 Gbaud QPSK, and an optical tone spaced by the desired $\mathrm{THz}$ frequency to be used as an optical carrier oscillator (LO). The delay between the two path lengths was carefully compensated in order to minimize the phase noise impact due to phase decorrelation between the unmodulated and modulated signals. Multi-channel $\mathrm{THz}$ wireless signals were generated by heterodyne

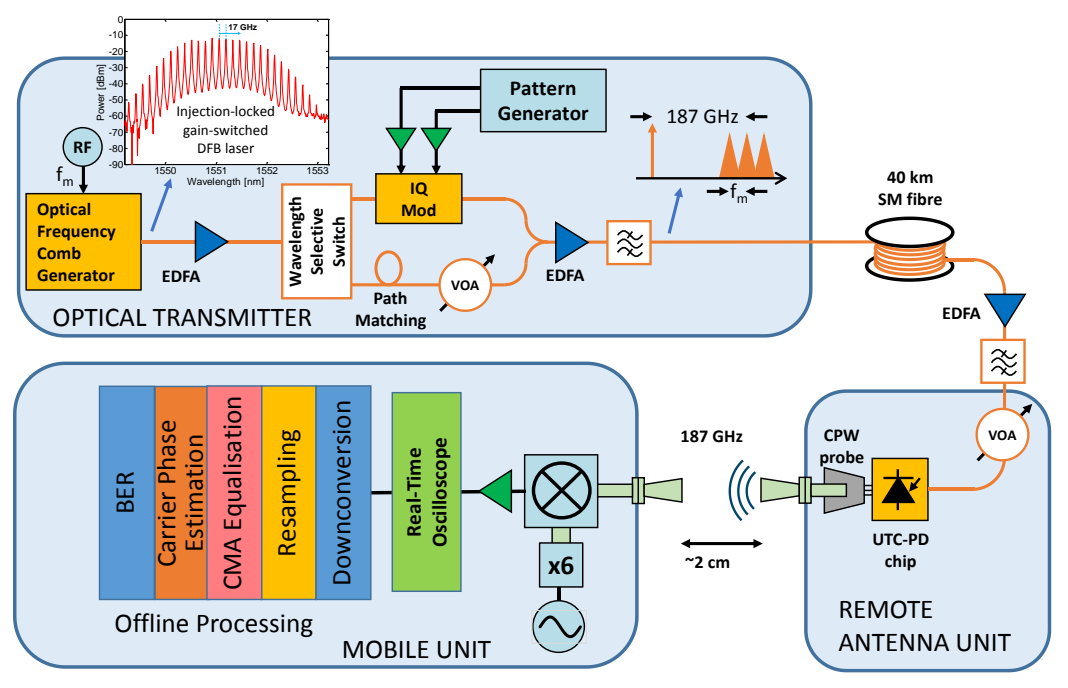

(a)

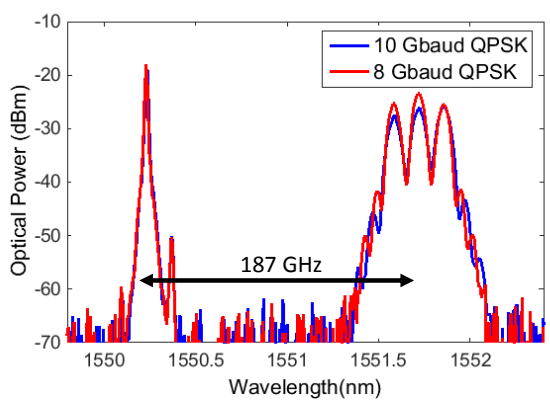

(b)

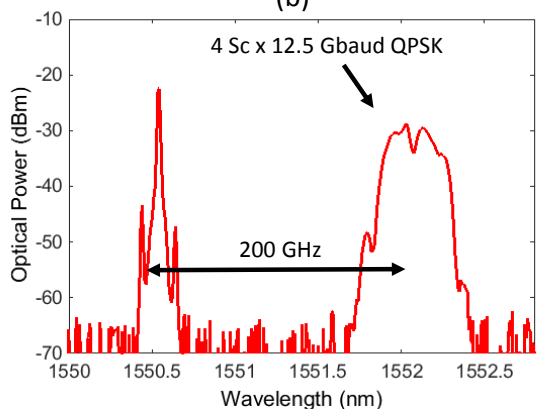

(c)

Figure 4. (a) Schematic diagram of photonic generation of THz wireless system using external injected gain-switched comb source, (b) and (c) the spectra for the modulated sub-carriers with 3Sc x QPSK at 8 and 10 Gbaud, and 4 SCs X 12.5 Gbaud.

mixing of the modulated QPSK optical carriers with one unmodulated optical tone spaced by about $200 \mathrm{GHz}$. Figure 4 (b) shows the spectra of the modulated optical subcarriers for three channels spaced by $17 \mathrm{GHz}$ and modulated with 8 or 10 Gbaud QPSK giving a total data rate of 48 or $60 \mathrm{Gbit} / \mathrm{s}$, and Figure 4(c) shows four channels spaced by $12.5 \mathrm{GHz}$ and modulated with 12.5 Gbaud QPSK for $100 \mathrm{Gbit} / \mathrm{s}$. The system showed an improvement in the generated $\mathrm{THz}$ frequency stability and phase noise performance, compared to the free running laser. This allows simplified DSP algorithms at the receiver, reducing the power dissipation and processing delays.

For short-range indoor link wireless $\mathrm{THz}$ distributions, we demonstrated experimentally photonic generation of multiband sub-THz wireless-over-fibre signals at up to $100 \mathrm{Gbit} / \mathrm{s}$ over the full band from $220-280 \mathrm{GHz}$ for the downlink stream, and $10 \mathrm{~Gb} / \mathrm{s}$ OOK for the uplink stream [18]. The experimental arrangement is shown in Figure 5. For the downlink stream, a single wavelength laser is used to generate coherent optical tones spaced by $15 \mathrm{GHz}$ using electro-optic modulation. These optical carriers are demultiplexed into odd and even groups and then separately modulated using two IQ modulators before they are recombined. The modulated signal was 10 Gbaud QPSK filtered by root raised cosine (RRC) with a rolloff factor 0.01 to limit the sub-bands to the Nyquist bandwidth. At the RAU, the optical multiband signal was optically filtered to select one of the data sub-bands and then combined with tunable laser (optical LO) with a linewidth of $100 \mathrm{kHz}$. 
A THz signal is generated with heterodyne mixing of the optical modulated signal and the optical LO. Then, this THz signal was radiated from a $20 \mathrm{dBi}$ antenna and received by the mobile unit, where it was downconverted and processed offline using DSP. Depending on the selected sub-THz band and the optical LO wavelength, the THz signal can be allocated on a different sub-THz band with frequency centered on 220, 235, 250, 265, or $280 \mathrm{GHz}$. For the uplink steam, the received $\mathrm{THz}$ is supposed to have been envelope detected and amplified before being used to modulate the same optical $\mathrm{LO}$ at the RAU. In this experiment, the uplink was implemented without the wireless channel for simplicity. Using this configuration, it allows the RAU to be reconfigured and enables frequency re-use capability by using a tunable LO laser. The system performance was determined by evaluating the BER versus the square of the photocurrent, which is expected to be proportional to the received $\mathrm{THz}$ power over a fixed wireless link length. The BER results were obtained for each of the sub-bands transmitted over the fiber independently, and then with the multiband transmitted. The BER results were well below $10^{-3}$ for the single sub-band, and an induced penalty was observed for the multiband due to the reduction of the optical power per sub-carrier after optical filtering. These measurements were conducted over a very short link $(\sim 2 \mathrm{~cm})$, however, by using polymethylpentene lenses with $50 \mathrm{~mm}$ diameter at both the transmitter and receiver, the transmission link was further extended to over $70 \mathrm{~cm}$ while the BER was kept well below the FEC limit.

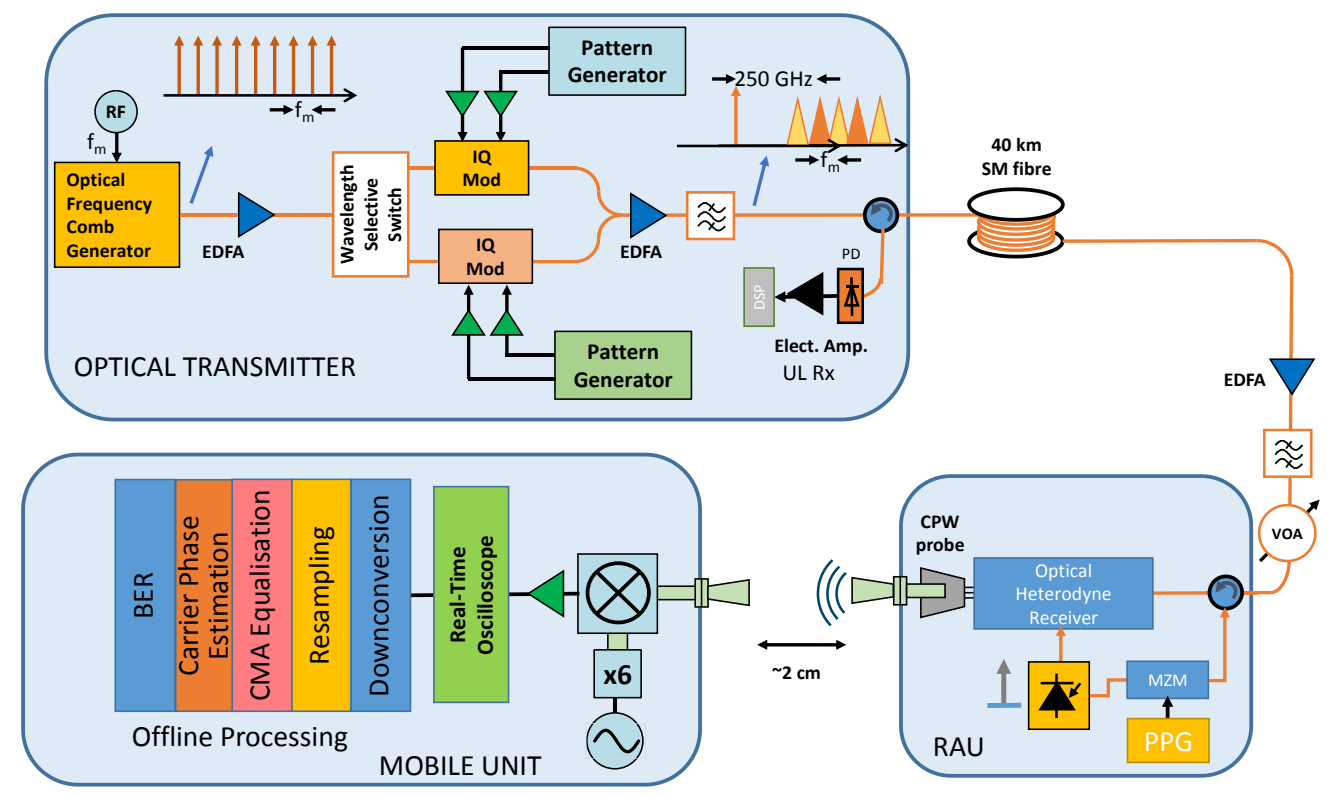

Figure 5. Schematic diagram for photonic multiband $\mathrm{THz}$ wireless system for uplink and downlink.

\section{CONCLUSIONS}

The integration of wireless-over-fiber with wireless $\mathrm{THz}$ is necessary for future extension of millimeter wave and $\mathrm{THz}$ signal distribution, in order to meet the future demands for high speed wireless communications. Photonic generation schemes for wireless $\mathrm{THz}$ have been reviewed based on using two free running lasers, and by using an optical frequency comb. The impact of the laser linewidths on the generated THz signal and on the performance of the system has been explored experimentally and simulated using Monte Carlo simulations. Photonic generation of multiband sub-THz bidirectional wireless-over-fiber communication for $100 \mathrm{Gbit} / \mathrm{s}$ aggregate downlink has been described, including provision of a $10 \mathrm{Gbit} / \mathrm{s}$ uplink optical stream. The main issue facing these photonic $\mathrm{THz}$ wireless systems is the lack of THz sources generating enough transmitted output power, which requires further integration between the UTC-PD and a power amplifier. In addition, implementing optical beamforming using phased array antennas could provide a solution to mitigate the high power loss in THz frequency by directing the power to mobile units. With these technological advances, practical systems for multi-Gbit/s wireless transmission at $\mathrm{THz}$ frequencies should become practical. 


\section{REFERENCES}

[1] S. Cherry, "Edholm's Law of Bandwidth - IEEE Spectrum," IEEE Spectr., vol. 41, no. 50, 2004.

[2] Cisco and/or its Affiliates, "Cisco Visual Networking Index : Global Mobile Data Traffic Forecast Update, 2015 $-2020, " 2016$.

[3] A. Seeds, H. Shams, M. Fice, and C. Renaud, "TeraHertz Photonics for Wireless Communications," J. Light. Technol., vol. 33, no. 3, pp. 1-1, 2014.

[4] H. Ito, F. Nakajima, T. Furuta, K. Yoshino, Y. Hirota, and T. Ishibashi, "Photonic terahertz-wave generation using antenna-integrated uni-travelling-carrier photodiode," Electron. Lett., vol. 39, no. 25, pp. 982-984, 2003.

[5] A. Beck, G. Ducournau, M. Zaknoune, E. Peytavit, T. Akalin, J. F. Lampin, F. Mollot, F. Hindle, C. Yang, and G. Mouret, "High-efficiency uni-travelling-carrier photomixer at $1.55 \mathrm{~mm}$ and spectroscopy application up to 1.4 THz.," Electron. Lett., vol. 44, no. 22, pp. 1320-1322, 2008.

[6] H. Ito, T. Furuta, F. Nakajima, K. Yoshino, and T. Ishibashi, "Photonic generation of continuous THz wave using uni-traveling-carrier photodiode," J. Light. Technol., vol. 23, no. 12, pp. 4016-4021, 2005.

[7] E. Rouvalis, C. C. Renaud, D. G. Moodie, M. J. Robertson, and A. J. Seeds, "Traveling-wave Uni-Traveling Carrier Photodiodes for continuous wave THz generation," Opt. Express, vol. 18, no. 11, pp. 11105-11110, 2010.

[8] E. Rouvalis, C. C. Renaud, D. G. Moodie, M. J. Robertson, and A. J. Seeds, "Continuous wave Terahertz generation from ultra-fast InP-based photodiodes," Microw. Theory Tech. IEEE Trans., vol. 60, no. 3, pp. 509$517,2012$.

[9] A. Hirata, T. Kosugi, H. Takahashi, J. Takeuchi, H. Togo, M. Yaita, N. Kukutsu, K. Aihara, K. Murata, Y. Sato, T. Nagatsuma, and Y. Kado, "120-GHz-band wireless link technologies for outdoor 10-Gbit/s data transmission," IEEE Trans. Microw. Theory Tech., vol. 60, no. 3 PART 2, pp. 881-895, 2012.

[10] H. Shams, M. J. Fice, K. Balakier, C. C. Renaud, and A. J. Seeds, "Multichannel 200GHz 40Gb / s Wireless Communication System using Photonic Signal Generation," pp. 4-7.

[11] A. Kanno, K. Inagaki, I. Morohashi, T. Sakamoto, T. Kuri, I. Hosako, T. Kawanishi, Y. Yoshida, and K. Kitayama, "20-Gb/s QPSK W-band (75-110GHz) wireless link in free space using radio-over-fiber technique," IEICE Electron. Express, vol. 8, no. 8, pp. 612-617, 2011.

[12] A. Kanno, K. Inagaki, I. Morohashi, T. Sakamoto, T. Kuri, I. Hosako, T. Kawanishi, Y. Yoshida, and K. Kitayama, "40 Gb/s W-band (75-110 GHz) 16-QAM radio-over-fiber signal generation and its wireless transmission.," in ECOC 2011 Technical Digest, 2011, vol. 19, no. 26, pp. B56-63.

[13] X. Pang, A. Caballero, A. Dogadaev, V. Arlunno, R. Borkowski, J. S. Pedersen, L. Deng, F. Karinou, F. Roubeau, D. Zibar, X. Yu, and I. T. Monroy, "100 Gbit/s hybrid optical fiber-wireless link in the W-band (75-110 GHz)," Opt. Express, vol. 19, no. 25, pp. 24944-9, Dec. 2011.

[14] L. Gonzalez-Guerrero, H. Shams, M. J. Fice, A. J. Seeds, and C. C. Renaud, "Linewidth Tolerance for THz Communication Systems Using Phase Estimation Algorithm," in Microwave Photonics (MWP), 2016 IEEE Topical Meeting on, 2016, pp. 1-4.

[15] "IEEE 802.15 WPAN Task Grooup 3d 100 Gbit/s Wireless (TG 3d (100G))," 2016. [Online]. Available: http://www.ieee802.org/15/pub/IGthz.html. [Accessed: 23-Nov-2016].

[16] H. Shams, M. J. Fice, K. Balakier, C. C. Renaud, F. van Dijk, and A. J. Seeds, "Photonic generation for multichannel THz wireless communication," Opt. Express, vol. 22, no. 19, pp. 23465-23472, Sep. 2014.

[17] H. Shams, T. Shao, M. J. Fice, P. M. Anandarajah, C. C. Renaud, F. Van Dijk, L. P. Barry, and A. J. Seeds, "100 $\mathrm{Gb} / \mathrm{s}$ Multicarrier THz Wireless Transmission System With High Frequency Stability Based on A Gain-Switched Laser Comb Source," IEEE Photonics J., vol. 7, no. 3, pp. 1-11, Jun. 2015.

[18] H. Shams, M. J. Fice, L. Gonzalez-Guerrero, C. C. Renaud, F. van Dijk, and A. J. Seeds, "Sub-THz Wireless Over Fiber for Frequency Band 220-280 GHz,” J. Light. Technol., vol. 34, no. 20, pp. 4786-4793, 2016. 